\title{
Interfacial Energy Measurement on Reconstructive Polymer Surface: Dynamic Polymer Brush by Segregation of Amphiphilic Block Copolymers
}

\author{
Masayuki Saito, ${ }^{\dagger}$ Norifumi L. Yamada, ${ }^{\ddagger}$, Kohzo Ito ${ }^{\dagger}$ and Hideaki Yokoyama*,†
}

†raduate School of Frontier Sciences, The University of Tokyo, 5-1-5 Kashiwano-ha, Kashiwa, Chiba 277-8561, Japan

\#Neutron Science Laboratory, High Energy Accelerator Research Organization, Ibaraki 319-1106, Japan

\section{Table of Contents}

1. Determination of Initial Size of Thin Films, $S_{\mathrm{i}} \mathrm{S} 2$ 


\section{Determination of Initial Size of Thin Films, $S_{\mathrm{i}}$}

Owing to the residual stress of spin-coated films, the initial size of thin films, $S_{\mathrm{i}}$, before deformation is not always the same as the mask size used in the reactive ion etching process. Therefore, we determined the initial size of thin films as follows.

Considering that stress $\sigma$ and strain $\lambda$ can be expressed as $\sigma=\Delta \gamma / d$ and $\lambda=\sqrt{S_{\mathrm{f}} / S_{\mathrm{i}}}$, eq. (2) can be rearranged as the following equation.

$$
\left(S_{\mathrm{f}} / S_{\mathrm{i}}\right)^{1 / 2}-\left(S_{\mathrm{f}} / S_{\mathrm{i}}\right)^{5 / 2}=3 \Delta \gamma / 2 d E \#(\mathrm{~S} 1)
$$

For neat SIS samples, $\Delta \gamma=\gamma_{\mathrm{L}}-\gamma_{\mathrm{S}}-\gamma_{\mathrm{SL}}(\approx 0.2 \mathrm{mN} / \mathrm{m})$ can be indirectly estimated by the general contact angle method $\left(\gamma_{\mathrm{L}}=72.4 \mathrm{mN} / \mathrm{m}, \gamma_{\mathrm{S}}=31 \mathrm{mN} / \mathrm{m}\right.$ and $\left.\gamma_{\mathrm{SL}}=41.6 \mathrm{mN} / \mathrm{m}\right)$. The thickness of thin films, $d$, and Young's modulus $E$ can be independently determined by ellipsometry and the tensile test of the bulk, respectively. The thicknesses, $d$ was in the range of $155-185 \mathrm{~nm}$, and Young's modulus $E$ of SIS was 1.0 MPa. Hence, $S_{\mathrm{i}}$ can be determined by eq. (S1) based on the measurement results of the areas of the neat SIS thin films floating on water. In this study, $S_{\mathrm{i}}$ was measured to be $233.4 \pm 1.6 \mathrm{~mm}^{2}$, and we used this value for calculating strain $\lambda$ to obtain the values of interfacial energy $\gamma_{\mathrm{SL}}$. 